This is a postprint version of the following published document:

Gowda, Apurva S.; Kazovsky, Leonid G.; Wang, Ke; Larrabeiti, David. (2015). Quasi-passive optical infrastructure for future $5 G$ wireless networks: pros and cons. Journal of optical communications and networking, 8(12), pp. B111-B123, 1 December 2016

DOI: https://doi.org/10.1364/JOCN.8.00B111

(C) 2016 Optical Society of America 


\title{
Quasi-Passive Optical Infrastructure for Future 5G Wireless Networks: Pros and Cons
}

\author{
Apurva S. Gowda, Leonid G. Kazovsky, Ke Wang, and David Larrabeiti
}

\begin{abstract}
In this paper, we study the applicability of the quasi-passive reconfigurable (QPAR) device, a special type of quasi-passive wavelength-selective switch with flexible power allocation properties and no power consumption in the steady state, to implement the concept of reconfigurable backhaul for $5 \mathrm{G}$ wireless networks. We first discuss the functionality of the QPAR node and its discrete component implementation, scalability, and performance. We present a novel multi-input QPAR structure and the pseudo-passive reconfigurable (PPAR) node, a device with the functionality of QPAR but that is pseudo-passive during steady-state operations. We then propose mesh and hierarchical backhaul network architectures for 5G based on the QPAR and PPAR nodes and discuss potential use cases. We compare the performance of a QPAR-based single-node architecture with state-of-the-art devices. We find that a QPAR node in a hierarchical network can reduce the average latency while extending the reach and quality of service of the network. However, due to the high insertion losses of the current QPAR design, some of these benefits are lost in practice. On the other hand, the PPAR node can realize the benefits practically and is the more energy-efficient solution for high reconfiguration frequencies, but the remote optical node will no longer be passive. In this paper, we discuss the potential benefits and issues with utilizing a QPAR in the optical infrastructure for 5G networks.
\end{abstract}

Index Terms Circuit-switched networks; Multicast network; Network topology; Optical devices; Packet-switched networks; Wavelength routing.

\section{INTRODUCTION}

$\mathbf{A}$ lot of research is being carried out on the design of optical backhaul networks for future cellular and WLAN Internet access at $\mathrm{Gb} / \mathrm{s}$ rates per user [1,2]. It is commonly agreed by the research community that 5G, the next generation of global cellular networks, should

Manuscript received June 1, 2016; revised October 20, 2016; accepted October 24, 2016; published November 23, 2016 (Doc. ID 266637).

A. S. Gowda and L. Kazovsky are part of the Photonics and Networking Research Lab (PNRL) in the Department of Electrical Engineering at Stanford University, California 94305, USA (e-mail: asgowda@stanford. edu).

K. Wang is with the Centre for Neural Engineering (CfNE), Department of Electrical and Electronic Engineering, University of Melbourne. He is also a visiting Assistant Professor in the Department of Electrical Engineering at Stanford University, California 94305, USA.

D. Larrabeiti is with the Telemetica Engineering Department at Universidad Carlos III de Madrid, Madrid, Spain. aim to provide tens of $\mathrm{Gb} / \mathrm{s}$ of peak data rates per user [3]. Recently, ITU R WP5D reached a global consensus on the key performance indicators (KPIs) targeted in 5G, including $20 \mathrm{~Gb} / \mathrm{s}$ peak data rate for low mobility users. This target rate is estimated to require $100 \mathrm{~Gb} / \mathrm{s}$ capacity for access, $\mathrm{Tb} / \mathrm{s}$ in the metro transport, and $\mathrm{Pb} / \mathrm{s}$ in the core transport. These access needs may be further increased if the cloud radio access network (C RAN) concept gets wide deployment in 5G. For example, a four sector $2 \times 2$ MIMO $20 \mathrm{MHz}$ LTE advanced base station (BS) requires a cumu lative bit rate of $9.83 \mathrm{Gbit} / \mathrm{s}$, according to the current CPRI specifications [4], which is $25 \%$ of the bit rate supported by NG PON2 (NG PON2 provides $4 \times 10 \mathrm{~Gb} / \mathrm{s}$ shared rates). Although different functional splits to reduce the fronthaul bit rate, such as the Next Generation Fronthaul Interface (NGFI) [5], are being studied, these fronthaul bit rates are being designed to be responsive to the payload data rate, which is expected to be $1000 \times$ that of $4 \mathrm{G}$ [3]. All these rates greatly exceed the possibilities of the current PON stan dards and call for the development of a more ambitious and versatile high capacity WDM backhaul system.

An option currently being explored to implement such a backhaul system is mmWave [6 8], where a large spectrum is available, and indeed, there are lab prototypes featuring multi Gbit/s links over distances of tens of kilometers. mmWave massive MIMO has also been proposed as a via ble backhaul technology between picocells and the macro BS in ultra dense networks [7]. However, the line of sight $(\mathrm{LoS})$ requirement of mmWave and its strong dependence on weather conditions [6] may limit the range of scenarios where this technology can be applied. In particular, achiev ing high bit rates becomes unpredictable in outdoor set tings, and inter BS communication is hard to achieve given the performance of this technology in point to multi point settings [9]. However, the rates required in the upper aggregation layer $(100 \mathrm{~Gb} / \mathrm{s}$ to $1 \mathrm{~Tb} / \mathrm{s})$ are only achievable with optical fiber technology, and hence the need to deploy very high density fiber access points with ultra high speed rates still remains. Additionally, the ideas of baseband unit (BBU) pools in central offices for ease of deployment and maintenance [10], packetization of the fronthaul (e.g., CPRI over Ethernet) [11,12] and fronthaul and backhaul packet multiplexing [13], impose strict latency and band width requirements on the access networks.

The ultra dense networks and high level of multiplexing for BS cooperation call for very large split ratios and the 
TABLE I

Novel Devices Versus State of the Art Devices

\begin{tabular}{lcll}
\hline Novel Device & Related State of the Art Device & Additional Functionality Over State of the Art & Insertion Loss \\
\hline QPAR & WSS & 1) Does not consume power in the steady state & QPAR $>$ WSS \\
& ROADM & 2) Variable power allocation to output ports & MI QPAR $>$ WSS \\
MI QPAR & WSS & Same as above & Same as QPAR but with low power consumption in steady state \\
PPAR & PPA $\Leftarrow$ WSS \\
\hline
\end{tabular}

support of a variety of topologies, ranging from cascaded trees to buses and rings, in optical access networks. In essence, the access network starts to adopt arbitrary shapes that evolve as the network and density grow. Since the transmission conditions and the traffic change as the physical network evolves, there will eventually be a need to re structure the topologies and capacities, prefer ably without manual intervention. Furthermore, since flex ibility implies the support of multiple paths through the network, the access network may start becoming a recon figurable mesh or ring with more than one connection to the core, following the natural evolution taking place in optical transport networks. Optical transport networks achieved this evolution by using the reconfigurable optical add drop multiplexer. We believe a quasi passive and re configurable (QPAR) device can bring about this evolution in access networks.

In this paper, we study the applicability of the QPAR de vice $[14,15]$ to implement this concept of reconfigurable backhaul for 5G wireless networks. We assume the sce nario in which BBU pools are located in the central office and optical access networks are used to transport fronthaul as well backhaul data over wide areas. In essence, a QPAR node has two main features: (1) splitting the input power into adjustable levels so as to share power only among a select number of ports, and (2) dynamic wavelength routing based on the bandwidth requirement. Additionally, the QPAR device does not consume power in the steady state and can be remotely powered for reconfiguration, thus avoiding on site maintenance [16]. Although originally, the device was designed for next generation PONs, in this paper, we explore scenarios where QPAR is used to gener ate arbitrary point to point or point to multipoint topol ogies among BSs. The use of QPAR in such networks requires upgrading the current single input QPAR node to multiple inputs. To that end, we present and analyze the novel multi input QPAR (MI QPAR) node. While the QPAR and MI QPAR nodes are quasi passive nodes with discrete power levels, another type of novel device called the pseudo passive reconfigurable (PPAR) device is pre sented and compared with the QPAR node. The PPAR node has the same functionality as the QPAR node and can sup port continuous power levels; however, it consumes a small amount of power in the steady state.

The paper is organized as follows: Section II describes the QPAR node and the previous work done on the node. Section III describes the implementation of the MI QPAR and PPAR nodes and their performances. Section IV describes the optical infrastructure options using QPAR for 5G, analyzes the performance, and briefly looks at the possibility of remote powering the optical wireless node. Section $\mathrm{V}$ discusses issues with real izing the 5G quasi passive reconfigurable backhaul, and, finally, we summarize the conclusions in Section VI.

\section{BACKGROUND}

In this section, previous studies on QPAR functions, principles, and implementations are briefly reviewed, since a similar approach is used in this paper to realize novel QPARs with multiple input ports, which provides better flexibility and can be used in more applications. Table I shows the new devices proposed compared with related state of the art devices.

The basic functionality of a QPAR node is shown in Fig. 1. A wavelength division multiplexing (WDM) signal with $N_{\lambda}$ wavelengths propagates through the QPAR node. It can be seen that QPAR enables dynamic signal unicast, multicast, and broadcast functions simultaneously. Importantly, the proposed QPAR device is capable of the multicast function to a configurable subset of output ports with variable power splitting ratios and without additional power losses. Unlike typical wavelength selective switches [17], power is reallocated, not blocked or lost.

In general, a QPAR node can be characterized by four dimensions: $N_{\text {in }} \times N_{\lambda} \times N_{p} \times N_{\text {out }}$, where $N_{\text {in }}$ is the number of input ports, $N_{\lambda}$ is the number of wavelengths, $N_{p}$ is the number of possible output power levels, and $N_{\text {out }}$ is the number of output ports. A power level here is defined as the fraction of the total transmission power assigned to each output port. The number of power levels is calculated from the full power to the smallest granularity (for simplic ity, the "zero" power level is not counted as a power level). The key enabling device in QPAR is the optical latching switch (OLS) [15], which typically can be realized using either micro electro mechanical systems (MEMS) or the opto magneto effect. In our demonstrations, the MEMS based OLSs are utilized due to their lower power consump tion. A QPAR with one input port has previously been demonstrated, and the general implementation principle

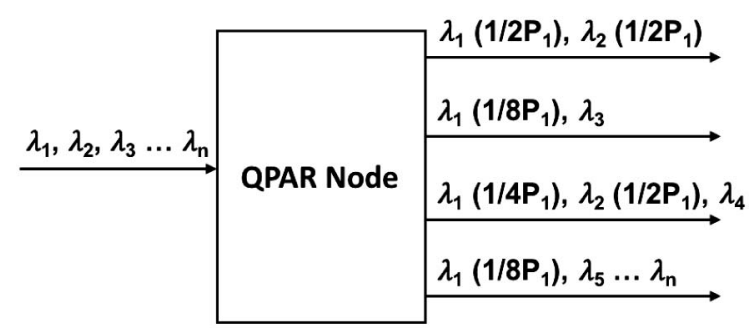

Fig. 1. QPAR device functionality: sample configuration. 


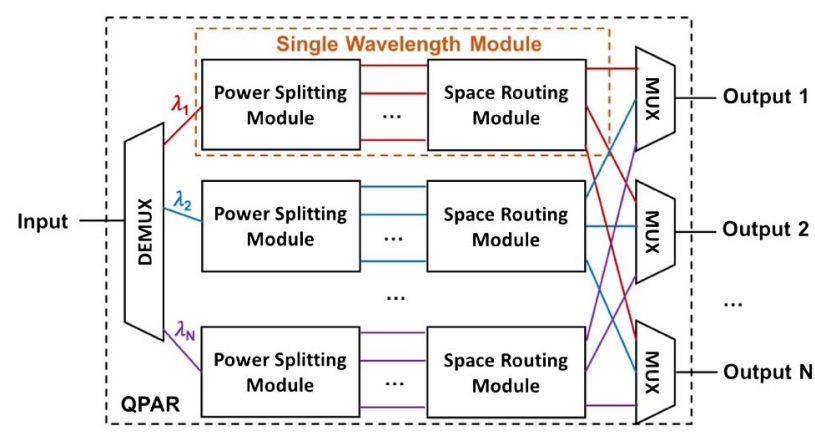

Fig. 2. Implementation principle of general QPAR nodes with a single input port.

is shown in Fig. 2 [15]. The performance and scaling of QPAR has also been studied in [15]; in this paper, we only focus on the proposed MI QPAR and PPAR nodes.

The QPAR node can also be operated for bidirectional signal transmissions as well by adding minimal extra com ponents (optical band filters or circulators) [18]. In the bi directional QPAR node, it is assumed that one downlink wavelength ( $\lambda_{1}$ and single wavelength module 1 ) is used to provide basic connections to all users, with more downlink wavelengths being added to provide higher speed connections according to user requirements. In the uplink direction, uplink signals bypass multiplexers/demulti plexers inside the QPAR with band filters or circulators, and all uplink signals propagate through single wave length module 1. Since the power distribution in the down link directions is typically proportional to the distances between the central office (CO) and optical network units (ONUs), uplink signals from different ONUs experience distance relevant power attenuations. As a result, uplink signals have similar power levels when reaching the $\mathrm{CO}$, and the power allocation can be dynamically varied. In ad dition, more wavelengths can be simply added to provide higher speed uplink data transmissions to end users, according to user specific requirements.

To completely eliminate local power supplies, two meth ods of remotely powering the QPAR node were developed and experimentally demonstrated [16]: direct photovoltaic power and charged supercapacitor power. A comparison be tween the two techniques shows that the direct photovol taic power option has better system energy efficiency and requires less time for reconfiguration. However, it requires a higher instantaneous power and thus, in an optical power limited condition, the charged supercapacitor power scheme is more suitable.

\section{MI-QPAR AND PPAR IMPLEMENTATION AND PERFORMANCE}

In this section, the implementation of QPAR with mul tiple input ports and the PPAR is discussed in detail; they provide better flexibility and can be used in more applications. The PPARs provide another option in fu ture networks requiring flexibility and reconfigurability, such as 5G networks. Therefore, the basic architecture, implementation, and operation principles are described in this paper. In addition to the QPAR node, an integrated pseudo passive reconfigurable node is proposed as well. Although the node is active, the power consumption is much lower than that of typical QPARs, and hence, it is more suitable for applications with frequent network reconfigurations.

\section{A. QPAR With Multiple Inputs}

To provide better flexibility and to be used in topologies such as mesh networks, a QPAR node with multiple input ports (MI QPAR) is needed to achieve quasi passive ROADM functionality. To provide the desired flexibility, the MI QPAR should be capable of dynamically distribut ing signals from any set of input ports to any set of output ports with variable wavelength and power combinations. Therefore, all input ports in MI QPAR are equivalent and interchangeable.

One possible MI QPAR structure is shown in Fig. 3. For each input port, the incoming signal is first demultiplexed and each wavelength passes through a single wavelength module, which consists of both the input and the output parts. The input and output modules are identical and con nected back to back. Inside the input module, variable power levels are generated, and they are dynamically guided toward destination output ports with OLSs and $3 \mathrm{~dB}$ optical couplers. Inside the output module, signals with the same wavelength from different input ports are dynamically combined before being multiplexed with sig nals at other wavelengths. In this way, signals from any input ports can be dynamically routed to any output ports with variable power levels and wavelength combinations.

\section{B. Pseudo-Passive Reconfigurable Node}

The proposed QPAR and MI QPAR nodes have the ad vantage of "quasi passive," which can significantly improve

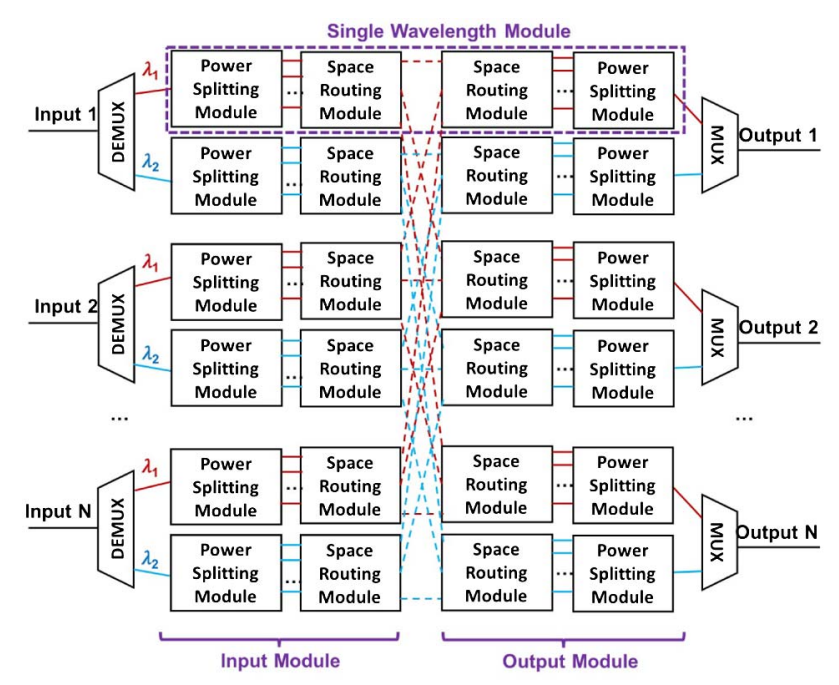

Fig. 3. Structure of the proposed MI QPAR node. 
TABLE II

Typical OLS Power Consumption

\begin{tabular}{lcc}
\hline OLS Dimension & $\begin{array}{c}\text { Typical Power } \\
\text { Consumption }(\mathrm{mW})\end{array}$ & $\begin{array}{c}\text { Typical Response } \\
\text { Time }(\mathrm{ms})\end{array}$ \\
\hline $2 \times 2$ & 525 & 0.41 \\
$1 \times 4$ & 530 & 2 \\
$1 \times 8$ & 40 & 2 \\
$1 \times 16$ & 150 & 5 \\
$4 \times 4$ & 125150 & 3.5 \\
\hline
\end{tabular}

the energy efficiency when the node is mostly operated under the steady state. However, when changes in the power and wavelength allocations are relatively frequent, the power consumptions of the QPAR and MI QPAR nodes are much higher, and they are no longer beneficial in terms of energy efficiency.

The typical power consumptions of MEMS based OLSs with different dimensions are shown in Table II. It can be seen that the power consumption for high dimension OLSs is greater than $100 \mathrm{~mW}$. As shown in Figs. 2 and 3, a large number of OLSs is needed when the QPAR or MI QPAR node has a large number of input ports, wavelengths, power levels, and output ports. Therefore, the large dimension node consumes relatively high power, and the energy consumption is also high when reconfigured fre quently. In this case, it can be more advantageous to use an all active (pseudo passive) reconfigurable node with low power consumption and an integrated design.

One possible integrated pseudo passive reconfigurable node with dynamic power and wavelength allocation capabilities based on the silicon photonics platform we pro posed is shown in Fig. 4. The integration of photonic devi ces on the silicon platform has been widely investigated during the past decade, mainly due to the possibility of us ing existing advanced and mature CMOS fabrication processes and facilities, which can enable low cost mass production. The proposed reconfigurable node is based on silicon micro ring resonators. The PPAR mainly consists of three parts, namely the input waveguide, micro rings for wavelength and power allocations, and output waveguides.

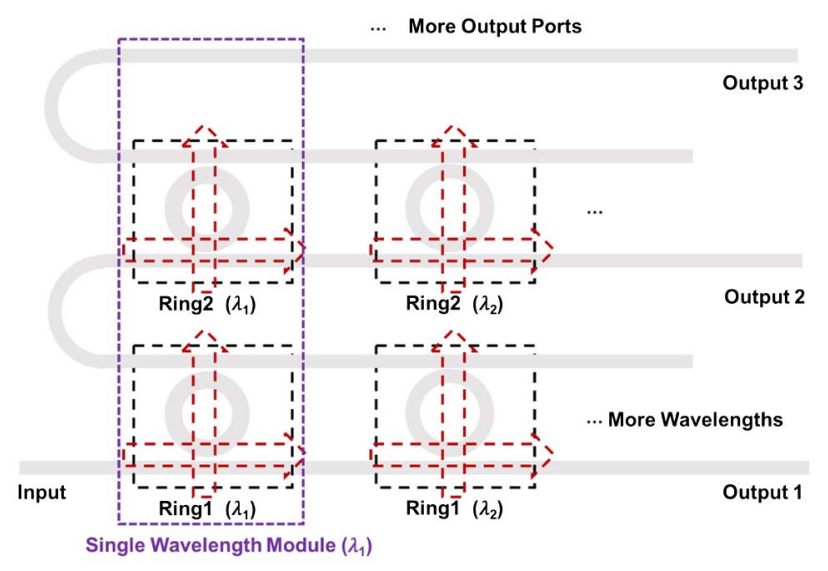

Fig. 4. Structure of the proposed pseudo passive reconfigurable node based on silicon integrated micro rings.
Micro rings with different resonant wavelengths are con nected in series along the signal propagation path to deal with signals at different wavelengths, and the resonant wavelength is controlled by choosing the appropriate ring radius. Several micro rings with the same radius are cas caded, constituting a single wavelength module. The num ber of rings needed in each single wavelength module is $N_{\text {out }}-1$, where $N_{\text {out }}$ is the number of output ports. By slightly changing the resonant wavelengths of these rings (e.g., thermal tuning), the power distribution to the output ports can be varied (different transmission/coupling ra tios). Through a similar wavelength tuning mechanism, the output signal wavelength combinations can be changed as well. Therefore, both the power and the wavelength can be dynamically allocated to the output waveguides/ports.

Compared with QPAR and MI QPAR nodes, the pseudo passive reconfigurable node based on silicon integrated micro rings have three major advantages. (1) The wave length multiplexing/de multiplexing and dynamic power allocation functions are realized simultaneously with the same device, i.e., a micro ring. Therefore, the complexity of the reconfigurable node can be reduced significantly for better scalability. (2) The power levels generated by the pseudo passive reconfigurable node are continuous and arbitrary, while the QPAR and MI QPAR nodes are only capable of generating a limited number of discrete power levels. Therefore, the pseudo passive reconfigurable node is capable of achieving better flexibility (which can be characterized by $N_{\text {in }} \times N_{\lambda} \times N_{\text {out }}$ ). (3) The pseudo passive reconfigurable node can be easily integrated, while it is challenging to realize integrated QPAR and MI QPAR nodes, mainly due to the OLSs. Magneto optic effect based OLSs face the limitations of material incompatibility and high scattering losses at material interfaces. MEMS based OLSs can be integrated on the silicon material; however, they have a non planar structure, which results in diffi culty integrating with other components, such as $3 \mathrm{~dB}$ op tical couplers. On the other hand, the major limitation of pseudo passive reconfigurable nodes compared with QPAR and MI QPAR nodes is the continuous power con sumption, as long as the power and wavelength allocation status are different from the initial design.

The silicon integrated micro rings can be tuned using the thermal optic effect, and the power consumption of thermal tuning can be extremely low, especially when the substrate directly beneath the micro rings is removed. Power consumptions as low as about $200 \mu \mathrm{W} / \mathrm{nm}$ have been experimentally demonstrated [19]. Since the tuning range required for dynamic power allocation is typically highly limited $(\ll 1 \mathrm{~nm})$, the realistic power consumption of each micro ring can be much lower than that of the OLS. To com pare the energy consumption of QPAR and pseudo passive reconfigurable nodes, here, $1 \times 4 \times 4 \times 4$ QPAR and $1 \times 4 \times 4$ pseudo passive reconfigurable nodes are consid ered. The simulation was carried out using Matlab. It was assumed that the QPAR node was reconfigured at time $=0 \mathrm{~s}$; then, it is operated in steady state. The PPAR was assumed to be reconfigured continuously during the period simulated. The maximum tuning range was considered to be $0.1 \mathrm{~nm}$, which is sufficient in typical 


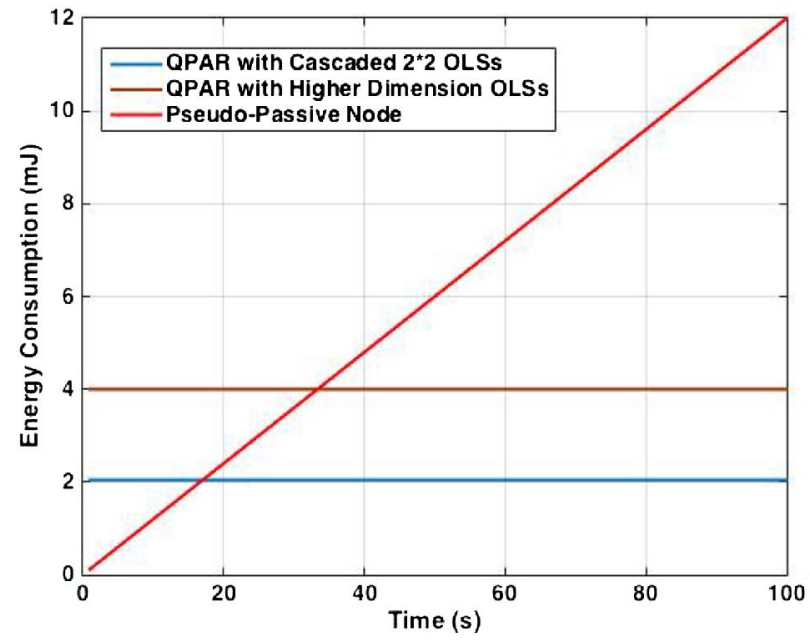

Fig. 5. Energy consumed during steady state by the $1 \times 4 \times 4 \times 4$ QPAR and $1 \times 4 \times 4$ pseudo passive reconfigurable nodes after reconfiguration at time $0 \mathrm{~s}$.

applications, and the tuning range follows a uniform distri bution. The total energy consumed by the QPAR and PPAR nodes is shown in Fig. 5. As discussed in the previous sec tion, QPAR can be scaled up in two ways, using either cas caded $2 \times 2$ OLSs or $4 \times 4$ OLSs, and both approaches are considered in the simulation. For the $2 \times 2$ and $4 \times 4$ OLSs, the power consumption is assumed to be the lower limit, i.e., 5 and $125 \mathrm{~mW}$, respectively, and the intermediate re sponse time is used, i.e., 0.7 and $4 \mathrm{~ms}$, respectively. It is clear from the figure that the QPAR node consumes energy due to the initial reconfiguration and then does not con sume any energy during steady state operations. On the other hand, the PPAR energy consumption increases lin early, even without any reconfiguration, due to the constant power consumption even in the steady state. In addition, the QPAR with cascaded $2 \times 2$ OLSs consumes less energy than the QPAR with $4 \times 4$ OLSs, which is con sistent with the power consumption and response time parameters shown in Table II. Importantly, it can be seen from the results that when the device reconfiguration is rel atively frequent $(<18 \mathrm{~s})$, the energy consumption of the pseudo passive node is even lower than that of the QPAR node. This implies that if reconfiguration occurs frequently, i.e., every $18 \mathrm{~s}$ or less, the QPAR energy con sumption, a staircase function with a step at every recon figuration, would be more than that of the PPAR node, a ramp function similar to the one depicted in Fig. 5. Thus, the PPAR node is more energy efficient when the reconfiguration frequency is high (cut off reconfiguration frequency is $55.56 \mathrm{mHz}$ in the simulation considered). It should be noted that in the analysis, the PPAR is capable of continuous power level variations, while the QPAR node is only capable of providing four possible power levels. When the available number of power levels increases, more $2 \times 2$ OLSs or higher dimension OLSs are needed; thus, the energy consumption of the QPAR node is higher, and the pseudo passive reconfigurable node cut off reconfigura tion frequency for energy efficiency is lower. A detailed comparison of these two approaches is outside of the scope of this paper.

\section{Performance and Scalability Analysis}

1) Performance of $Q P A R$ and MI QPAR: To provide better flexibility and to support other network topologies, a MI QPAR node was proposed in the previous section. To investigate the performance of the proposed MI QPAR node, here we consider a MI QPAR device with five input ports and five output ports. One possible application sce nario of such a device is in the mesh based 5G mobile op tical WDM backhaul network elaborated on in Section IV. Possible application scenarios we envision are quick hand overs where the MI QPAR is used to form point to point dedicated optical connections between base stations, and CoMP, where a base station needs to form a point to multipoint connection to multiple base stations.

In the simulation, the total number of wavelengths available in the network varies between 5 and 100. Each base station is assumed to try to use a random wavelength to form a point to point or point to multipoint connection with another random base station or stations. The traffic arrival rate from the input port (i.e., a base station) is $R$ requests/s, following a Poisson distribution. The traffics generated from different input ports are independent. The destination output ports (i.e., destination base stations) are also considered to be random, and each signal can be guided to a random number of output ports (for multipoint connections). The traffic lengths are assumed to be uni formly distributed between 0.1 and $4 \mathrm{~s}$. The simulation parameters are summarized in Table III.

Inside the MI QPAR node, traffics arriving at the same time instance from different input ports with the same wavelength may collide inside the single wavelength mod ules. In this case, the colliding traffics are considered to be blocked, and the MI QPAR node performance is analyzed using the blocking probability parameter. The simulation results for the blocking probability at different numbers of available wavelengths are shown in Fig. 6. The traffic arrival rate changes between 0.1 to 1 request/s. In each simulation, a total of $2500 \mathrm{~s}$ was considered. Ten rounds of simulations were carried out under each scenario, and the average results are shown.

From the simulation results, it can be seen that the block ing probability is higher when the total number of wave lengths available is small. This is mainly due to the increased possibility that the traffics generated in different directions use the same wavelength. Furthermore, it is clear that a higher traffic arrival rate also results in a higher

TABLE III

Simulation Parameters

\begin{tabular}{lcc}
\hline Simulation Type & $\begin{array}{c}\text { Number of Input } \\
\text { Ports }\end{array}$ & $\begin{array}{c}\text { Number of Output } \\
\text { Ports }\end{array}$ \\
\hline Blocking Probability & 5 & 5 \\
Scalability & 5 & 5,6 , or 8 \\
\hline
\end{tabular}




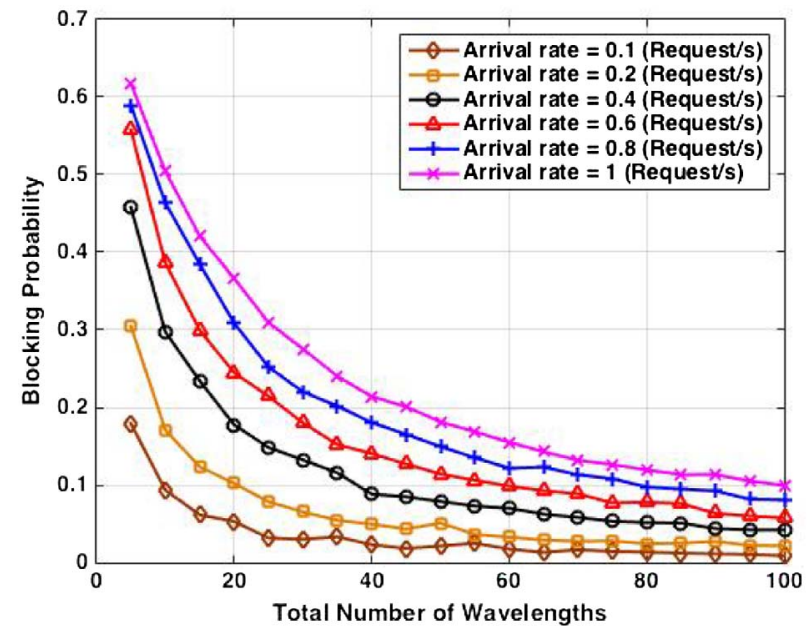

Fig. 6. Simulation results on the blocking probability of the MI QPAR node with five input ports.

blocking probability. These phenomena are mainly due to the higher probability that signals with the same wavelength ar rive at the MI QPAR node simultaneously, which potentially leads to collisions in single wavelength modules.

It should be noted that the traffic blocking probability here is considerably high. This is mainly due to two rea sons: (1) the signal wavelength assignment in the simula tions is completely random for all input ports. In reality, wavelength assignment and control protocols and algo rithms are always used, which can significantly reduce the node blocking probability (one simple example is serving the blocked traffic with a number of sharing wave lengths (pre determined and fixed) without the optical shortcut (i.e., flow service) to ensure basic connections); and (2) in the simulations, flow traffic is used and the length of the traffic is considerably long (up to $4 \mathrm{~s}$ ), which leads to the substantially high probability that a new flow session is initiated and arrives at the MI QPAR node before the previous flow session completely propagates through the node. When the traffic length is shorter, the blocking probability can be considerably lower.

2) Scalability: In addition to the blocking probability, the scalability of the QPAR node is also an important parameter, especially for low cost considerations. The scal ability of QPARs with a single input port has been previ ously analyzed [15]. Therefore, here we only focus on the scalability analysis of MI QPAR nodes.

As discussed in the previous section, the dimension of the MI QPAR nodes can be described as $N_{\text {in }} \times$ $N_{\lambda} \times N_{p} \times N_{\text {out }}$. For MI QPAR with structures as shown in Fig. 3, the number of single wavelength modules needed is $N_{\text {in }} \times N_{\lambda}$, since each input port has a full set of single wavelength modules. As shown in [15], two methods can be used to scale up the number of power levels and output ports supported by the single wavelength modules. Using the first scaling up solution with cascaded bi state ele ments (each consisting of a $2 \times 2$ OLS and a $3 \mathrm{~dB}$ optical coupler), the number of bi state elements $N_{B}$ needed in each single wavelength is

$$
N_{B}=\left\{\begin{array}{cc}
2\left(2^{N_{p}-1}-1\right) & \text { if } N_{p} \leq \log _{2} N_{\text {out }}+1 \\
N_{\text {out }}\left(N_{p}-\log _{2} N_{\text {out }}\right) & \text { if } \log _{2} N_{\text {out }}+1 \leq N_{p} \\
-N_{\text {out }}+2\left(N_{\text {out }}-1\right) & \leq N_{\text {out }}
\end{array}\right.
$$

In addition to the bi state elements, two additional $N_{\text {out }} \times N_{\text {out }}$ OLSs are needed in each single wavelength module for the space routing function. If the second scal ing up solution is used (based on using higher dimension OLSs for dynamic power splitting), in each single wave length module, two $2 N_{\text {out }} \times 2 N_{\text {out }}$ OLSs, two $N_{\text {out }} \times N_{\text {out }}$ OLSs, and $N_{p}-13 \mathrm{~dB}$ optical couplers are needed. If $\log _{2} N_{\text {out }}+1 \geq N_{p}$, the $2 N_{\text {out }} \times 2 N_{\text {out }}$ OLSs can be replaced by $N_{\text {out }} \times N_{\text {out }}$ OLSs. Based on the above analysis, the num ber of components (OLSs and $3 \mathrm{~dB}$ optical couplers) in the MI QPAR nodes is calculated, and the results are shown in Fig. 7. The number of input ports is fixed at five and both scale up solutions are considered. It is clear that the num ber of components needed is always larger when scale up solution 1 (cascaded bi state elements) is used, especially

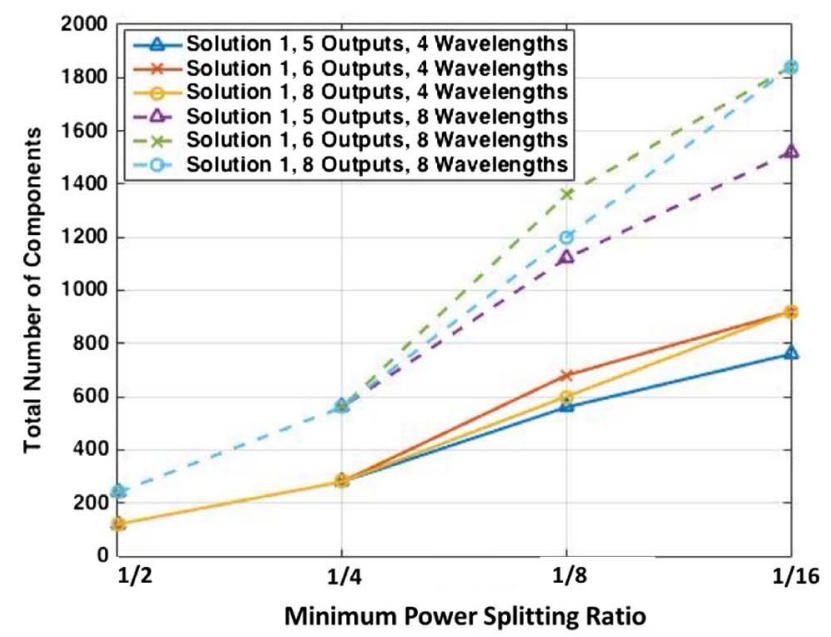

(a)

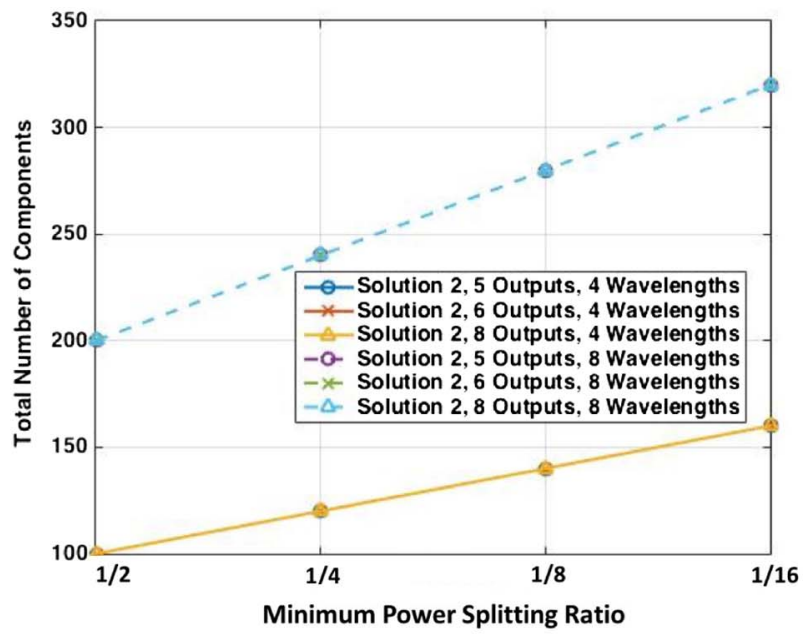

(b)

Fig. 7. Number of components (OLS and $3 \mathrm{~dB}$ optical couplers) needed in MI QPAR with 5 input ports. (a) With scale up solution 1 , and (b) with scale up solution 2 . 
when the number of power levels supported by MI QPAR nodes is large. It can also be seen that the number of com ponents needed increases linearly with the number of wavelengths. Furthermore, for MI QPARs using scale up solution 1, the number of components required also in creases when the number of output ports is higher. On the other hand, the number of components remains con stant when scale up solution 2 is used. However, it should be noted that higher dimension OLSs $\left(2 N_{\text {out }} \times 2 N_{\text {out }}\right)$ are needed in solution 2 for the dynamic power allocation. As shown by the results in Fig. 7, the number of compo nents needed in the proposed MI QPAR is comparatively large, which results in node complexity and cost issues. These issues can be solved by using either tri state ele ments or through the photonic integration of OLSs with $3 \mathrm{~dB}$ optical couplers, as discussed in detail in [15].

\section{QPAR IN 5G BACKHAUL NeTWORKS}

As discussed in the introduction, 5G backhaul networks need to be flexible, dynamic, reliable, and able to support aggregate bit rates over 100 Gbps. The previous sections highlight the flexibility of QPAR in terms of wavelength and power allocation as well as reconfigurability. In this section, we discuss the network architectures that utilize QPAR and the potential advantages and 5G use cases for such networks. We also compare the latency of fixed networks versus reconfigurable unicast networks and mul ticast networks. The unicast network can employ a passive splitter or a unicast wavelength selective switch (WSS) or a QPAR with the power allocation property unused. The mul ticast network can be realized using a passive splitter, a multicast WSS, or a QPAR. Finally, we discuss the advan tages of flexible power allocation supported by QPAR when compared to a colorless passive splitter or multicast WSS. The PPARs proposed in the previous section can also be used in the network scenarios discussed in the following subsections, where QPARs are just replaced by PPARs. Therefore, the network performances with PPARs are also analyzed and compared.

\section{A. Proposed 5G Network Architectures}

In this section, we propose $5 \mathrm{G}$ backhaul architectures that utilize QPAR and discuss the potential use cases in a $5 \mathrm{G}$ network. If we look at the evolution in the metro and core networks, it is clear that the network became more flexible and achieved higher capacities by evolving from point to point to ring and mesh topologies. Future $5 \mathrm{G}$ networks will require low latency connections between base stations for quick handovers, cooperative communica tions (CoMP), etc. To this end, a reconfigurable mesh top ology that allows any base station to set up a point to point connection to any other base station or multiple base sta tions would be beneficial. An example of quick handovers would be for vehicular networks or high mobility applica tions where handovers occur frequently and latency during handovers needs to be very low. In such scenarios, the ability to quickly form point to point connections at the

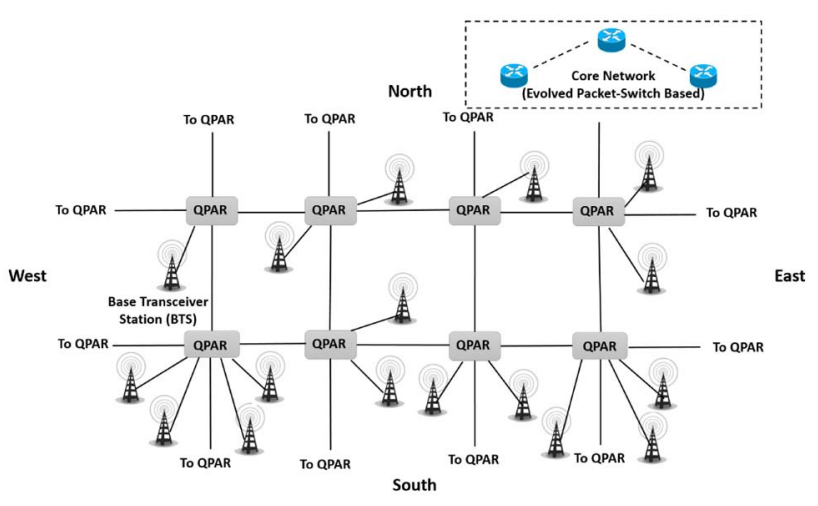

Fig. 8. $5 \mathrm{G}$ mesh network with QPAR.

physical layer can go a long way. Another potential use case would be for cooperative networks when multiple base sta tions need to share their channel and user information. The mesh topology, depicted in Fig. 8, utilizes a MI QPAR at every splitting node, which can be used to route wave lengths from any base station to any other base station or multiple base stations using the flexible power alloca tion property of QPARs and thus can form point to point/ point to multipoint connections at the physical layer be tween base stations. As shown in Section III, the probabil ity of a connection being blocked depends on the number of wavelengths available and the duration of the connection. It should be noted that the blocking probability presented here is for a single node, and in a multi hop scenario, the blocking probability would increase due to the wavelength continuity constraint. However, for the scenarios described above, more often than not, the connection is with a neigh boring base station and is thus a single hop. As mentioned, a wavelength assignment algorithm and control protocol to coordinate the connections or the use of a fixed set of shared wavelengths can reduce the blocking probability. Since the QPAR node can be remotely reconfigured and powered (see Section II), a centralized control algorithm can easily be used. A mesh topology has the added benefit of a high number of redundant paths for recovery and traffic engineering.

While the mesh topology is attractive for its flexibility and high number of redundant paths, the deployment can be expensive, and in practice, the network may be under utilized. Figure 9 shows an alternative hierarchical network. In this network, the capacity ( $\lambda$ 's and $N_{p}$ ) of the QPAR nodes is gradually increased from the edge to the core. All QPAR nodes have at least two disjoint paths to the core for fault protection. If so decided, the edge of the network can be based on an unprotected PON architec ture with a single input QPAR connected to multiple base stations through a single port via a passive splitter. This type of network is more conducive to traffic aggregation and distribution, as most of the backhaul traffic is meant to reach the core. Point to point connections between base stations can be supported using the intra PON QPAR setup that is described in [20]. Thus, redundant routes and topol ogies are available; however, the latency may be affected since the traffic has to go up a layer and then back down. 


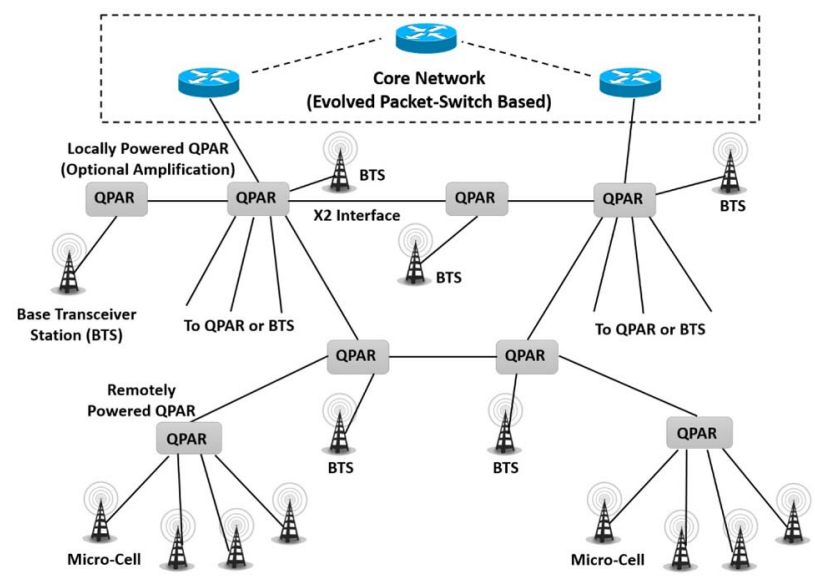

Fig. 9. 5G hierarchical network using QPAR.

In this section, we assess the latency improvements of reconfigurable wavelength allocation to base stations and then compare the different methods to realize this scheme in a network from the optical power budget perspective.

\section{B. Simulation Scenario}

The advantage of point to point dedicated optical cir cuits between base stations and the core in terms of latency is clear. Therefore, we focus on the more cost effective ex ploitation of a shared PON where wavelengths are shared by multiple base stations for packet switched backhauling. We simulate a simple traffic engineering scenario for a TWDM PON, which is used to provide backhaul to multiple base stations (Fig. 10). The packets being transported could be fronthaul or backhaul data (BBU pools located at the central office). This is to help evaluate the latency and power improvements that can be realized by utilizing a QPAR node in the current standardized PON used for 5G backhauling. TWDM PON is a multicast network pro tocol, since a single wavelength can be shared amongst multiple, though not necessarily all, ONUs being served by the OLT. However, in the traditional passive splitter based network architecture, all wavelengths are broadcast

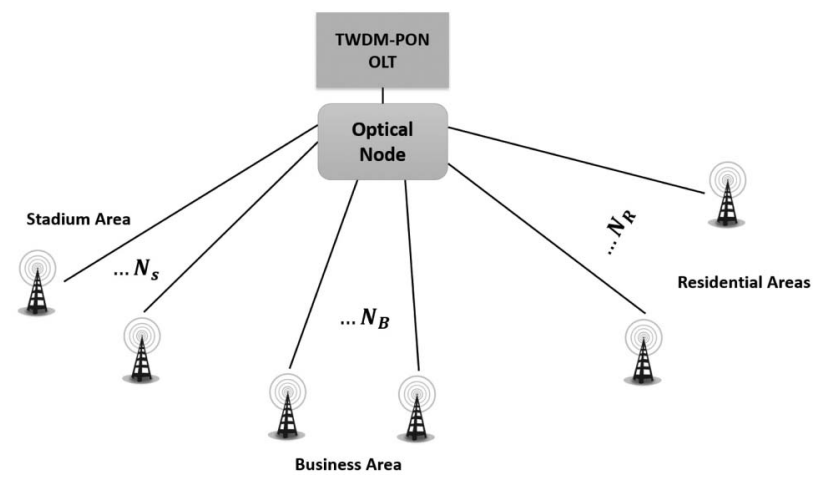

Fig. 10. Simulation scenario. to all ONUs, which would require the ONUs to possess a tunable filter. With a multicast network architecture real ized using a WSS or a QPAR, the appropriate wavelengths can be directed to the appropriate ONUs and the ONUs can be simple fixed receivers. The network aims to serve three different zones: a business area, a residential area, and a stadium area, as shown in the figure. The type of device to be used as an "optical node" in Fig. 10 depends on the flex ibility level. We assume that the supporting network archi tecture can have three levels of flexibility in terms of wavelength allocation:

1. Fixed wavelength allocation to zones: Wavelength allo cation to different zones is decided at the time of deploy ment. The network is easily realized using a passive splitter and fixed filters. The base stations would have fixed transceivers.

2. Reconfigurable wavelength allocation to zones: A wave length can be assigned to one zone only, but it can be reconfigured. One way to realize the optical splitting node is by using a $1 \times 3$ WSS with each port connected to a passive splitter. In this way, all the power is shared amongst the base stations in a zone. Another option is to use the QPAR, leaving the dynamic power allocation capability unused. The simplest method is a passive splitter.

3. Reconfigurable wavelength allocation to base stations: Wavelengths are assigned per base station; as such, they can be assigned to a zone, portions of a zone, or span multiple zones. In this case, the optical node can be a passive splitter, a multicast WSS, or a QPAR.

In all cases, we assume that a base station can transmit and receive packets on one wavelength at a time. An impor tant point to note is that the passive splitter, being color less, splits all the wavelengths to all the base stations; thus, each base station would be required to have a tunable transceiver to tune to the allotted wavelength and tune out the rest of the wavelengths. In the WSS and QPAR case, the wavelengths can be selectively routed to ports; thus, the base stations need only be fixed transceivers, pro vided that the wavelengths used are within their optical bandwidth. The OLT would reconfigure the node to route the allotted wavelength to the appropriate port. This would eliminate the need for costly tunable transceivers at every base station.

We simulate a downlink scenario for typical TWDM PON parameters, i.e., assuming four wavelengths and a total of 64 base stations. We assume that the division of base stations among the zones (stadium, business, resi dential) is $(24,24,16)$. For the fixed wavelength allocation, we assign one wavelength to each zone, and the fourth wavelength is assigned to the business area (premium service). For the reconfigurable wavelength allocation to zones, one wavelength is guaranteed to each zone, and the extra wavelength is assigned to the zone with the high est load. Within that zone, the load is evenly distributed among the wavelengths. For the reconfigurable wavelength allocation to base stations, the total load of the network is evenly distributed among the wavelengths. The traffic into 
the OLT is assumed to be Poisson and the peak data rate is limited to $100 \mathrm{Gbps}$ (projected access network capacity for 5G [3]) with a leaky bucket shaper. The data rate supported by each wavelength is assumed to be $10 \mathrm{Gbps}$. The packets are assumed to be between 1000B and 1500B (uniformly distributed). We choose large packet sizes since the major ity of current LTE backhaul packets are of a large size due to high video, HTTP, and FTP traffic [21]. The traffic is as sumed to be 5 million requests/s, such that on average, all the wavelengths would be overloaded. The OLT is assumed to have a infinite buffer, and we calculate the average la tency experienced by the base stations over a $10 \mathrm{~ms}$ trans mission window. We consider such an overloaded scenario to better perform a comparative analysis of the three set ups in terms of latency improvements. The wavelength allocation is done based on the loads in different zones, which we assume vary at different times of the day.

The reconfiguration times for a multicast WSS and a QPAR are on the same order, between hundreds of micro seconds to a few milliseconds. For the traffic engineering scenario considered, the load change (and thus the recon figuration) occurs on a much larger scale (a few minutes to a few hours). Thus, the overall effect of the reconfiguration time on the latency becomes negligible. Similarly, the tun ing time of the transceivers is in the order of a few micro seconds, which is also negligible compared to the frequency of reconfiguration. Thus, in our study, we only consider the time between the packet arriving at the OLT and the time it reaches its destination.

The gains evaluated in this study are for a single node in the network. Multi node network simulations to compare the MI QPAR in the mesh and hierarchical topologies are being conducted for subsequent publications.

1) Queuing Latency: Figure 11 shows the average packet transport latency for different times of day. During work hours, i.e., 9 am $5 \mathrm{pm}, 50 \%$ or $70 \%$ of the load is assumed to be for the business area. Similarly, in the eve ning hours, $50 \%$ or $70 \%$ of the load originates from the res idential areas, and during game time, $50 \%$ or $70 \%$ of load is assumed to originate from the stadium area. We see that over any time of day and with medium or large imbalances in load, reconfigurable wavelength allocation to base sta tions has a low average latency. We can also see that with this scheme, the variance in latency between different times of day, as well as between medium and large unbal ances in load, is relatively small (latency varies by about $2 \mu \mathrm{s}$ in the multicast network, while it varies by an order of 100 times when the imbalance in the load becomes too large in the unicast network). This is essentially due to the maximum flexibility available to distribute the load among the wavelengths.

Simulation results for the upstream scenario can be obtained by adding an additional latency to these results to represent resource access control delays.

We also simulate the scenario where inter BS communi cation is supported for applications such as CoMP. We com pare two scenarios: Bi QPAR as the optical node, shown in Fig. 10, and MI QPAR as the optical node. In the case of

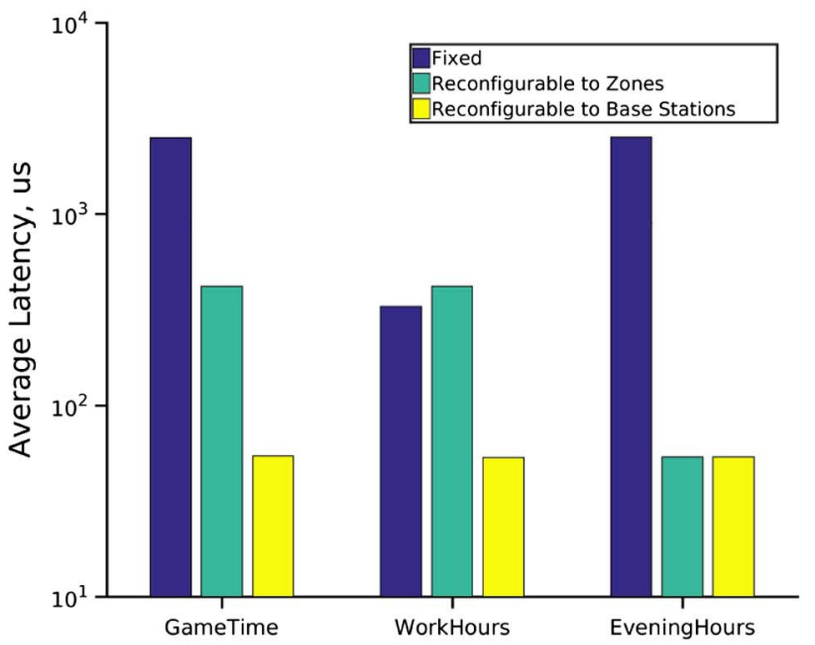

(a)

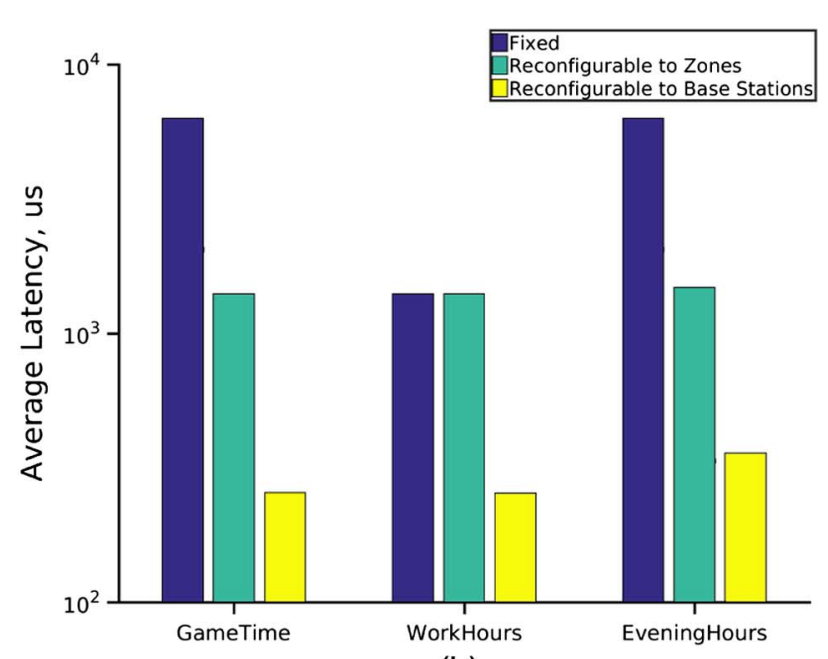

(b)

Fig. 11. Average queuing latency during different times of day for the fixed, unicast, and multicast networks. (a) $50 \%$ of the load to single zone, and (b) $70 \%$ of the load to a single zone.

Bi QPAR, the payload would have to traverse to the OLT and be re routed back to the appropriate BSs. With MI QPAR, the payload can be directly routed to the appropriate BS or BSs (point to multipoint). The only limit posed by the device to the frequency of forming a point to multipoint con nection is its response time, i.e., the time duration from the change of the applied electrical control signal to the change of the optical signal. For the designed QPAR device utilizing $2 \times 2$ OLSs, the response time is a few hundred microseconds. The PPAR device can support high reconfiguration frequen cies and is the more energy efficient solution in such cases.

In the simulations, we assume the BS communication data is uniformly distributed between $0.11 \mathrm{~ms}$ and is be tween BSs in the stadium area. In these simulations, we assume point to point BS communication though point to multipoint communication is supported. We consider the game time scenario with $70 \%$ of the total downlink load destined to the stadium area. The total downlink load is 


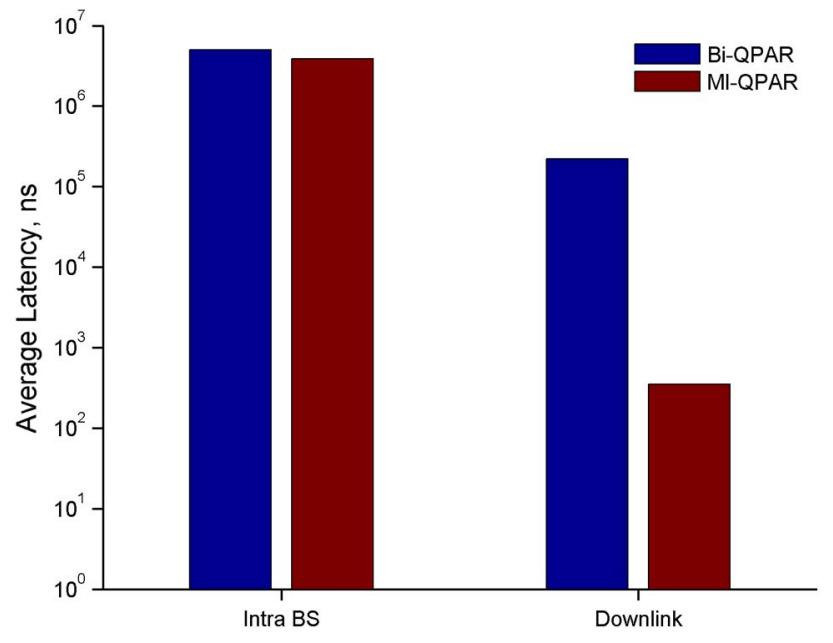

Fig. 12. Average queuing latency assuming inter BS communi cations.

assumed to be $40 \%$. No priority is assumed for the intra BS communications. For the case of Bi QPAR, we assume the intra BS communication is in the form of Ethernet packet sizes. Figure 12 shows the average latency for the intra BS and downlink packets. We see that using the MI QPAR, the intra BS latency is lower and, more importantly, the down link packets have an average latency two orders of magni tude lower since the downlink data does not include the inter BS communication.

2) Power: From the previous section, we see that the multicast network is the clear choice for low latency. However, which of the three nodes, i.e., passive splitter, multicast WSS, or QPAR/PPAR, is the most advantageous remains unanswered. In this section, we compare the three nodes from the optical power budget perspective. We assume the same scenario as described in the previous section and calculate the maximum distance that can be supported by the node for a given optical power budget, $P_{\text {budget }}$.

The optical power budget is given as below:

$$
P_{\text {budget }}=\alpha D+\mathrm{SL}+\mathrm{IL}, \mathrm{dB},
$$

where $\alpha$ is the optical fiber loss in $\mathrm{dB}$ per unit distance, $D$ is the distance of the base station from the optical node, SL is the splitting loss in $\mathrm{dB}$, and IL is the insertion loss in $\mathrm{dB}$. We assume an optical power budget of $32 \mathrm{~dB}$, which is for class $C+$ optical transceivers. For the passive splitter and the multicast WSS, the splitting loss depends on the num ber of output ports of the node, which, in our simulations, is 64. For the QPAR and the PPAR, the splitting loss depends on the power allocated by the node to the particular output port. In our analysis, we calculate the minimum fraction of power required by the base station based on its distance from the optical node. We then sum the fractions of power allocated to all the base stations. If the sum is less than one, the base station distribution sample can be supported, i.e., it is a feasible solution. If the sum is greater than one, the sample is infeasible. If any base station is too far such that the optical power budget is not sufficient to support the distance, the sample is infeasible. Since the PPAR can support continuous power levels, the minimum fraction of power required by base station $i$ is

$$
r_{i}=\mathrm{SL}_{i}=P_{\text {budget }}-\alpha D_{i}-\mathrm{IL}, \mathrm{dB} .
$$

For the discrete level QPAR design presented in the pre vious section, the minimum power allocation required is $r_{i}=1 / 2^{N_{\mathrm{QPAR}}}$, where $N_{\mathrm{QPAR}}=\min \left\{\left\lfloor\log _{2}\left(10^{\mathrm{SL}_{i} / 10}\right)\right\rfloor, N_{\min }\right\}$. Here, $N_{\min }$ is the minimum power level supported by the QPAR node.

We generate 500 base station distribution samples over a circular area, assuming a uniform distribution over the area and a non uniform distribution with a higher density of base stations closer to the optical node. Figure 13 shows the maximum distance for which all the generated base station distribution samples are feasible for an ideal node with no insertion loss. We calculate the maximum distance for the different number of users per wavelength. In the cases of QPAR and PPAR, the lower the number of base stations per wavelength, the higher the fraction of power available to each base station. Thus, we see that as the number of users per wavelength decreases, the maximum distance that can be supported by QPAR and PPAR in creases. This is not the case for the passive splitter or multi cast WSS, where power cannot be reallocated because of its constant splitting ratio and, consequently, a fixed maximum deployment distance. From the load scenarios considered in the latency simulations, the number of base stations allot ted the same wavelength is always less than 64 . We also see that the QPAR and PPAR are more beneficial when the base station distribution is denser near the optical node (QPAR and PPAR can support $20 \%$ higher deployment distance, even for 64 users). PPAR can provide the most increase in deployment distance, between $20 \% 80 \%$, depending on the maximum number of base stations that are likely to be as signed the same wavelength. The use of discrete power levels in the current design of QPAR slightly reduces the gain. A point to note is that for the distances shown, in the cases

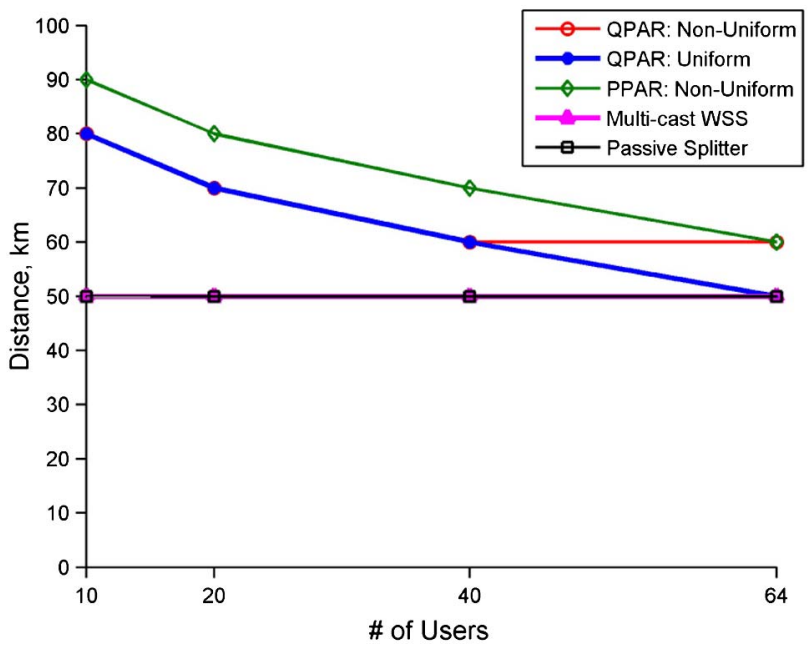

Fig. 13. Maximum distance supported versus number of base stations with no insertion loss. 


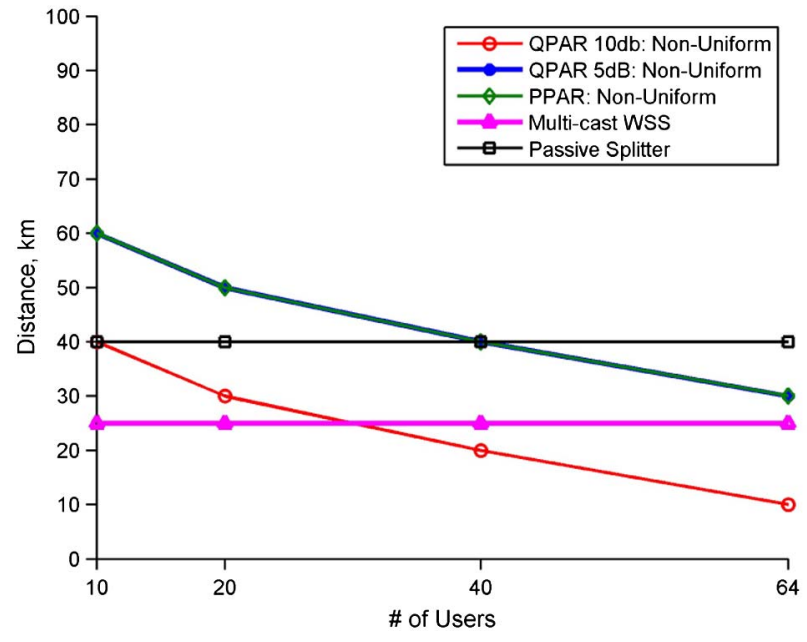

Fig. 14. Maximum distance supported versus number of base stations with insertion loss.

of QPAR and PPAR, on average, about $30 \% 40 \%$ of the total power is unallocated. This extra power can be allocated to select base stations to reduce the bit error rate and improve their quality of service and reliability.

Figure 14 shows the maximum distance supported by the nodes, including insertion loss. The insertion loss of a passive splitter is around $3 \mathrm{~dB}$, while that of a multicast WSS is around $56 \mathrm{~dB}$ [22], which reduces the maximum deployment distances to $\sim 40$ and $\sim 25 \mathrm{~km}$, respectively. The current implementation of QPAR has a very high insertion loss (an implementation with tri state MEMs has an inser tion loss of about $10 \mathrm{~dB}$ for 64 outputs), so we see it cannot support $40 \mathrm{~km}$ for more than 10 users per wavelength. However, it does support a larger distance than the multi cast WSS for at least 20 base stations per wavelength. The PPAR has a slightly better insertion loss, $7 \mathrm{~dB}$ for 64 output ports, and thus, we can see gains of up to $20 \%$ compared to a passive splitter. To see the same gain with QPAR, the in sertion loss has to be $5 \mathrm{~dB}$.

The flexibility in power reallocation provided by QPAR and PPAR is a bonus in terms of distance and quality of service per base station. However, in the case of QPAR, these bonuses can be practically realized only if the insertion loss is less than about $5 \mathrm{~dB}$. The current discrete component de sign has a high insertion loss and cannot outperform the passive splitter. However, a more integrated QPAR design may be able to realize a lower insertion loss. The PPAR pro posed in this paper and experimentally demonstrated can practically realize power reallocation benefits and outper forms the multicast WSS. Although unlike the passive split ter, the optical node is active during the steady state, the power consumed is low and it thus can be powered remotely.

\section{Remotely Powered Optical/Wireless Node: Is It Doable?}

In the mesh topology, a wireless node can be co located with the QPAR. Since the QPAR is powered remotely, we consider a scenario where the whole node, QPAR + wire less, is powered remotely. This would eliminate the need for a local power supply and, more importantly, allow the wireless node to be easily controlled centrally for energy efficiency purposes, i.e., we can turn off the wireless node in the absence of users. Several studies have shown that the wireless section of the network is the most power consuming, so such a simple way to control sleep mode would go a long way.

While the concept is attractive, implementation is very challenging because of the high power consumed by the wireless node. If we consider the simplest scenario of RF signal distribution, i.e., analog radio over fiber, over a small cell $(<20 \mathrm{~m})$, the main power consuming component would be the power amplifier. For $20 \mathrm{~m}$, the output power would need to be around $15 \mathrm{dBm}$ (this is the output power of a typical Internet of things WLAN module). If we assume a 30\% drain efficiency, the power consumed by the power amplifier would be around $P_{\text {out }} / 0.3=105.4 \mathrm{~mW}$. Assuming the drain voltage is $5 \mathrm{~V}$, the current that needs to be deliv ered to the power amplifier would be $\sim 21 \mathrm{~mA}$. Applying the direct photovoltaic power scheme described in Section II, the power to each PD in an eight in series InGaAs PD array (used in the experiments in [16]) would have to be around $16 \mathrm{dBm}$; thus, the total optical power that would be needed to be delivered to the $\mathrm{PD}$ array would be $\sim 25 \mathrm{dBm}$, which is very challenging. For a macro cell covering a cell of a few kilometers in radius, the output power alone can be in the range of $>20 \mathrm{~W}$, which results in a power amplifier drain current of $13.4 \mathrm{~A}$, which is not feasible using the remote powering techniques. Thus, we can see that even for the simplest scenario, i.e., only a power amplifier in a small cell, remote powering the wireless node is difficult. However, this is a preliminary analysis based on compo nents used in a laboratory experiment, and we are contin uing to look at other options of realizing such a remotely powered optical wireless node.

\section{QPAR IN 5G BACKHAUL ARCHITECTURES: PROS AND Cons}

A QPAR node in an optical infrastructure can provide benefits in terms of latency and variance in latency, which is important for $5 \mathrm{G}$ wireless networks. In essence, the adoption of QPAR nodes in optical access networks can re duce the latency by allowing flexible network topologies that allow for smart dynamic bandwidth allocation proto cols. This reduction in latency would pave the way to easier fronthaul and backhaul packet integration in optical access networks. Additionally, it can increase the deployment dis tance and/or the quality of service to base stations using the flexible power allocation capability. Using the MI QPAR in a mesh or hierarchical topology, one can easily form point to point or point to multipoint connections for low latency applications such as quick handovers, CoMP, etc. The use of QPAR has the added benefit of sharing the power only among the required number of ports. Due to the remote reconfigurability possible with the QPAR node, all this flexibility can be realized with minimal 
maintenance at the optical nodes. Also, with the QPAR node, reconfigurable wavelength allocation can be done with fixed transceivers at the base stations instead of tunable transceivers.

While ideally, the QPAR device is beneficial, the primary drawback of the current discrete component design is the insertion loss and scalability of the devices. With the current practical QPARs, the previously discussed advantage of ex tended network reach, or equivalently, the larger number of end users that can be supported by the network, is difficult to achieve [15]. The comparatively high insertion loss of QPARs is mainly due to device limitations. QPARs are based on OLSs and $3 \mathrm{~dB}$ power splitters, and the OLSs used in our designs were MEMS based, which had comparatively large losses. Furthermore, the coupling loss between adjacent components also results in high insertion loss in the QPARs. As discussed in [15], with other advanced types of OLSs and the promising and rapidly developing photonic integration technologies, the QPAR insertion losses can be significantly reduced. It should be noted that the major advantages of the flexible wavelength and power allocation functions of QPARs can always be achieved, regardless of the device insertion loss. We also would like to point out that other types of reconfigurable nodes, such as those based on WSSs, also have higher insertion losses compared with fixed solutions. Compared with these reconfigurable nodes, QPARs have the advantage of being "quasi passive," where no power is needed during steady state operations. Therefore, the network energy efficiency can be improved substantially. A more integrated QPAR design with a lower insertion loss $(\sim 5 \mathrm{~dB})$ can realize these benefits; however, several challenges exist in the integration of MEMs based OLSs having non planar structures with $3 \mathrm{~dB}$ optical cou plers and other components that have planar structures. Further research in this area is needed. Using the inte grated PPAR device, some of the benefits can be practically realized despite the higher insertion loss and can be scaled to a higher dimension easily. However, the remote node is no longer passive in the steady state. Remote powering using the techniques mentioned in the previous section is a pos sibility due to the low power consumption.

The reconfiguration time of the QPAR node is around a few hundred microseconds to a few milliseconds. For the traffic engineering scenario considered, reconfiguration may be done every few hours, in which case, such reconfig uration times are acceptable. However, for high mobility handovers, the reconfiguration may be done every few sec onds, in which case, the current MEMs based QPAR recon figuration time could be an issue. The PPAR has a much lower reconfiguration time, of the order of tens to a few hundred microseconds, and as such, may be a better solu tion for networks with high reconfiguration frequencies. Also, as shown, the PPAR is the more energy efficient sol ution when the reconfiguration frequency is high.

The greater the flexibility, the greater the amount of con trol required in the network. With densely deployed QPARs, wavelength and power assignment control algo rithms and protocols would be needed. Since the QPAR and PPAR nodes can be remotely configured, a centralized control algorithm can be deployed. This is another open area for research.

\section{Conclusions}

In this paper, we present the potential of a QPAR device in 5G backhaul networks. We extend the discrete compo nent implementation of the single input QPAR device to multiple inputs, i.e., multi input QPAR (MI QPAR), a quasi passive alternative to ROADMs with flexible power distribution control and wavelength routing. The blocking probability of the node depends on the number of available wavelengths, duration of the connection, and number of ports. In practice, a wavelength allocation and control scheme could limit blocking. Also presented is a pseudo passive reconfigurable node, which has an integrated de sign and the added advantage of continuous output power levels. Unlike QPAR, PPAR consumes power in the steady state; however, we show that for high reconfiguration frequencies, the PPAR node is more energy efficient. At the network level, we propose two network architectures, mesh and hierarchical, based on the QPAR node and dis cuss potential application scenarios. We show that the QPAR node in a shared hierarchical packet switched net work (e.g., PON) reduces the latency under unbalanced loads. Using the MI QPAR node significantly reduces the latency for inter BS communication. We also show that the network reach can be increased using the dynamic power allocation property. However, to practically achieve these improvements, the insertion loss of QPAR has to be less than at least $5 \mathrm{~dB}$. Though the current discrete component design of QPAR has a higher insertion loss than $5 \mathrm{~dB}$, a more integrated design may be able to achieve $a \leq 5 \mathrm{~dB}$ insertion loss. On the other hand, the integrated PPAR design presented in this paper has a low enough insertion loss to realize the gains from flexible power and wave length allocation, but the remote node is no longer passive. Nevertheless, the PPAR node can be remotely powered due to the low steady state power consumption. We believe the potential gains in networks from an integrated QPAR design in terms of flexibility and maintenance warrant fur ther research in this area.

\section{ACKNOWLEDGMENT}

This work has been funded by the Spanish project TIGRE5 CM (grant number S2013/ICE 2919), the EU H2020 5G Crosshaul project (grant number 671598), and the Australian Research Council's Discovery Early Career Researcher Award (DECRA) funding scheme (project number DE150100924). The authors would also like to acknowledge the support of the Center for Integrated Systems, Stanford University, and Corning Incorporated for the development of this work.

\section{REFERENCES}

[1] T. Pfeiffer, "Next generation mobile fronthaul and midhaul architectures [Invited]," J. Opt. Commun. Netw., vol. 7, no. 11, pp. B38 B45, 2015. 
[2] 5G PPP Architecture Working Group, "View on 5G architec ture," July 2016 [Online]. Available: https://5g ppp.eu/ wp content/uploads/2014/02/5G PPP 5G Architecture WP For public consultation.pdf.

[3] J. G. Andrews, S. Buzzi, W. Choi, S. V. Hanly, A. Lozano, C. K. Soong, and J. C. Zhang, "What will 5G be?" IEEE J. Sel. Areas Commun., vol. 32, no. 6, pp. 1065 1082, 2014.

[4] A. de la Oliva, J. A. Hernandez, D. Larrabeiti, and A. Azcorra, "An overview of the CPRI specification and its application to C RAN based LTE scenarios," IEEE Commun. Mag., vol. 54, no. 2, pp. 152 159, Feb. 2016.

[5] China Mobile Research Institute, "White paper of next gen eration fronthaul interface," Oct. 2015 [Online]. Available: http://labs.chinamobile.com/cran/wp content/uploads/2015/09/ NGFI Whitepaper EN v1.0 201509291.pdf.

[6] Z. Pi and F. Khan, "An introduction to millimeter wave mobile broadband systems," IEEE Commun. Mag., vol. 49, no. 6, pp. 101 107, 2011.

[7] Z. Gao, L. Dai, D. Mi, Z. Wang, M. A. Imran, and M. Z. Shakir, "MmWave massive MIMO based wireless backhaul for the 5G ultra dense network," IEEE Wireless Commun., vol. 22, no. 5, pp. 13 21, 2015.

[8] W. Feng, Y. Li, D. Jin, L. Su, and S. Chen, "Millimetre wave backhaul for 5G networks: Challenges and solutions," Sensors, vol. 16, pp. 892 908, June 2016.

[9] R. Taori and A. Sridharan, "Point to multipoint in band mmwave backhaul for 5G networks," IEEE Commun. Mag., vol. 53, no. 1, pp. 195 201, 2015.

[10] D. Wubben, P. Rost, J. S. Bartelt, M. Lalam, V. Savin, M. Gorgoglione, A. Dekorsy, and G. Fettweis, "Benefits and im pact of cloud computing on $5 \mathrm{G}$ signal processing: Flexible cen tralization through cloud RAN," IEEE Signal Process. Mag., vol. 31, no. 6, pp. 35 44, 2014.

[11] N. J. Gomes, P. Chanclou, P. Turnbull, A. Magee, and V. Jungnickel, "Fronthaul evolution: From CPRI to Ethernet," Opt. Fiber Technol., vol. 26, pp. 50 58, Dec. 2015.

[12] L. Valcarenghi, K. Kondepu, and P. Castoldi, "Analytical and experimental evaluation of CPRI over Ethernet dynamic rate reconfiguration," in IEEE Int. Conf. on Commun. (ICC), Kuala Lumpur, 2016, pp. 16.

[13] A. D. La Oliva, X. C. Perez, A. Azcorra, A. Di, Giglio, F. Cavaliere, D. Tiegelbekkers, J. Lessmann, T. Haustein,
A. Mourad, and P. Iovanna, "Xhaul: Toward an integrated fronthaul/backhaul architecture in 5G networks," IEEE Wireless Commun., vol. 22, no. 5, pp. 32 40, 2015.

[14] Y. Bi, J. Jin, and L. G. Kazovsky, "Quasi passive reconfigur able optical node: First experimental demonstration," in Conf. on Lasers and Electro Optics (CLEO), 2012, paper CTh1H.5.

[15] Y. Bi, J. Jin, A. R. Dhaini, and L. G. Kazovsky, "QPAR: A quasi passive reconfigurable green node for dynamic resource management in optical access networks," J. Lightwave Technol., vol. 32, no. 6, pp. 1104 1115, 2014.

[16] Y. Bi, S. Shen, J. Jin, K. Wang, and L. G. Kazovsky, "Remotely powered and reconfigured quasi passive reconfigurable nodes for optical access networks," J. Electr. Comput. Eng., vol. 2016, $2938415,2016$.

[17] T. A. Strasser and J. L. Wagener, "Wavelength selective switches for ROADM applications," IEEE J. Sel. Top. Quantum Electron., vol. 16, no. 5, pp. 1150 1157, 2010.

[18] K. Wang, Y. Bi, and L. G. Kazovsky, "Bidirectional quasi passive reconfigurable (Bi QPAR) node for flexible access networks," in Conf. on Lasers and Electro Optics (CLEO), San Jose, CA, 2016, paper JTh2A.123.

[19] P. Dong, W. Qian, H. Liang, R. Shafiiha, D. Feng, G. Li, J. E. Cunningham, A. V. Krishnamoorthy, and M. Asghari, "Thermally tunable silicon racetrack resonators with ultralow tuning power," Opt. Express, vol. 18, no. 19, pp. 20298 20304, 2010.

[20] S. Yin, S. Shen, Y. Bi, J. Jin, T. Oyama, and L. G. Kazovsky, "A novel quasi passive, software defined, and energy efficient optical access network for adaptive intra PON flow transmis sion," J. Lightwave Technol., vol. 33, no. 22, pp. 4536 4546, 2015.

[21] NGMN Alliance, "Backhaul provisioning for LTE advanced \& small cells," Dec. 2014 [Online]. Available: https://www.ngmn. org/uploads/media/150929 NGMN P SmallCells Backhaul for LTE Advanced and Small Cells.pdf.

[22] B. Robertson, H. Yang, M. M. Redmond, N. Collings, J. R. Moore, J. Liu, A. M. Jeziorska Chapman, M. Pivnenko, S. Lee, A. Wonfor, I. H. White, W. A. Crossland, and D. P. $\mathrm{Chu}$, "Demonstration of multi casting in a $1 \times 9$ LCOS wave length selective switch," J. Lightwave Technol., vol. 32, no. 3 , pp. 402 410, 2014. 